\title{
A mutation in $\beta$-amyloid precursor protein renders SH-SY5Y cells vulnerable to isoflurane toxicity: The role of inositol 1,4,5-trisphosphate receptors
}

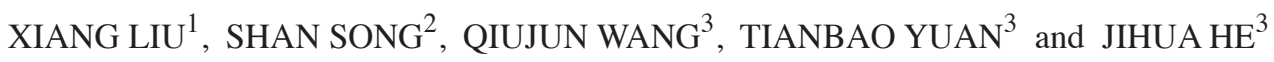 \\ ${ }^{1}$ Department of Anesthesiology, Children's Hospital of Hebei Province, Shijiazhuang, Hebei 050031; \\ ${ }^{2}$ Department of Anesthesiology, Yantai Yu Huang Ding Hospital, Yantai, Shandong 264000; \\ ${ }^{3}$ Department of Anesthesiology, The Third Hospital of Hebei Medical University, Shijiazhuang, Hebei 050051, P.R. China
}

Received July 14, 2015; Accepted August 1, 2016

DOI: $10.3892 / \mathrm{mmr} .2016 .5930$

\begin{abstract}
Isoflurane is a commonly used inhaled anesthetic, which induces apoptosis of SH-SY5Y cells in a dose- and time-dependent manner; however, the underlying mechanisms remain unknown. The authors of the present study hypothesized that a mutation in $\beta$-amyloid precursor protein (APP), which is a gene associated with familial Alzheimer's disease, may render cells vulnerable to isoflurane-induced cytotoxicity via activation of inositol 1,4,5-trisphosphate receptors (IP3R). In the present study, SH-SY5Y cells were transfected with a vector or with mutated APP, and were treated with the equivalent of 1 minimum alveolar concentration (MAC) isoflurane for $8 \mathrm{~h}$. Cell apoptosis rate, alterations to cytosolic calcium concentrations $\left(\left[\mathrm{Ca}^{2+}\right]_{\mathrm{c}}\right)$, and protein levels of IP3R were determined following exposure of cells to isoflurane. In addition, the effects of the IP3R antagonist xestospongin $\mathrm{C}$ were determined on isoflurane-induced cytotoxicity and calcium release from the endoplasmic reticulum (ER) of mutated APP- and vector-transfected SH-SY5Y cells. Treatment with isoflurane (1 MAC) for $8 \mathrm{~h}$ induced a higher degree of cytotoxicity, and a marked increase in $\left[\mathrm{Ca}^{2+}\right]_{\mathrm{c}}$ and IP3R protein levels in mutated APP-transfected SH-SY5Y cells compared with vector-transfected SH-SY5Y cells. Xestospongin C significantly attenuated isoflurane-mediated cytotoxicity and inhibited calcium release from the ER of SH-SY5Y cells. These results indicated that the APP mutation may render SH-SY5Y cells vulnerable to isoflurane neurotoxicity, and the underlying mechanism may be associated with $\mathrm{Ca}^{2+}$ dysregulation via overactivation of IP3R.
\end{abstract}

Correspondence to: Professor Qiujun Wang, Department of Anesthesiology, The Third Hospital of Hebei Medical University, 139 Ziqiang Road, Shijiazhuang, Hebei 050051, P.R. China E-mail: 18032288937@163.com

Key words: isoflurane, cells, inositol 1,4,5-trisphosphate receptors, apoptosis

\section{Introduction}

Cognitive decline may occur following major surgery and anesthesia, and has been reported by patients and their families for decades. Elderly patients are particularly susceptible to such an event, which is known as postoperative cognitive dysfunction (POCD). POCD is self-limiting in the majority of patients (1); however, in some patients it may be long-term or permanent. Previous studies have reported that $25 \%$ of elderly patients exhibit POCD 1 week following non-cardiac surgery, whereas $\sim 10 \%$ of elderly patients exhibit POCD 3 months after non-cardiac surgery $(2,3)$; however, the association with general anesthesia remains unclear. Inhalational anesthetics, including isoflurane, sevoflurane and desflurane, are generally considered to be safe in clinical anesthesia; however, numerous studies have demonstrated that these agents can induce cell damage, neurodegeneration or POCD (4-8). These observations raise concerns regarding the possibly deleterious effects of general anesthesia in elderly patients.

Isoflurane is a common inhalational anesthetic agent, exposure to which may induce cytotoxicity in various neuronal and non-neuronal tissues and cells. In addition, isoflurane has been reported to trigger widespread neuronal apoptosis in the developing rat brain, subsequently leading to persistent learning deficits and cognitive dysfunction, which may persist for several weeks following treatment in adults, and aged rats and mice $(2,3)$. Our previous study demonstrated that exposure to isoflurane at 1 minimal alveolar concentration (MAC) for $12 \mathrm{~h}$, or at $2 \mathrm{MAC}$ for $8 \mathrm{~h}$, may decrease cell viability, and these effects may be associated with the disruption of intracellular calcium homeostasis (9). Intracellular calcium homeostasis is primarily regulated by three protein receptors on the endoplasmic reticulum (ER): Inositol 1,4,5-trisphosphate receptors (IP3R), ryanodine receptor (RyR) and $\mathrm{Ca}^{2+}$-ATPases $(2,3)$, IP3R on the ER membrane is able to induce non-physiological calcium release, thus leading to a depletion of ER calcium, and increased cytosolic $\left(\left[\mathrm{Ca}^{2+}\right]_{c}\right)$ and mitochondrial calcium; these effects may contribute to cell apoptosis (10). A presenilin-1 mutation associated with familial Alzheimer's disease (AD) has been reported to render neurons vulnerable to isoflurane toxicity, via the induction of abnormal calcium release from 
the ER through IP3R activation (8). Mutations in $\beta$-amyloid $(A \beta)$ precursor protein (APP) are also associated with AD; therefore, the present study hypothesized that this mutation may increase cell susceptibility to isoflurane-induced cytotoxicity.

The aim of the present study was to clarify whether the APP mutation enhances susceptibility to isoflurane-mediated apoptosis, and whether this effect was induced by $\mathrm{Ca}^{2+}$ dysregulation via IP3R overactivation.

\section{Materials and methods}

Cell culture. SH-SY5Y neuroblastoma cells can undergo neuronal maturation and have been previously used as an in vitro cell model for studying the mechanisms of neuronal differentiation and neurotoxicity. The SH-SY5Y human neuroblastoma cell line was obtained from the Shanghai Institute for Biological Sciences of the Chinese Academy of Sciences (Shanghai, China) and were cultured in Dulbecco's' modified Eagle's medium (DMEM; Sigma-Aldrich; Merck Millipore, Darmstadt, Germany) supplemented with $10 \%$ fetal bovine serum (Sigma-Aldrich; Merck Millipore), $200 \mu \mathrm{g} / \mathrm{ml} \mathrm{G} 418,100 \mathrm{U} / \mathrm{ml}$ penicillin and $100 \mu \mathrm{g} / \mathrm{ml}$ streptomycin. Monolayer cultures at a density of $0.3 \times 10^{5}$ cells $/ \mathrm{cm}^{2}$ were incubated in plastic flasks in a humidified atmosphere containing $95 \%$ air and $5 \% \mathrm{CO}_{2}$ at $37^{\circ} \mathrm{C}$. The medium was changed every 2 days, and cells were passaged once they had reached 70-80\% confluence. When the SH-SY5Y cells reached $70 \%$ confluence, the cells were transfected with overexpression plasmid pcDNA3.1-APP695, containing a mutant APP695 gene (Wanleibio, Shenyang, China) using the Lipofectamine 2000 reagent (Invitrogen; Thermo Fisher Scientific, Inc., Waltham, MA, USA) according to the manufacturer's instructions. Cells were transfected with vector alone or mutated APP. Prior to isoflurane exposure, medium was replaced with serum-free DMEM.

Anesthetic exposure. Cells grown in plastic flasks were exposed to isoflurane (1 MAC; $8 \mathrm{~h}$ ) in a gas-tight chamber inside a cell culture incubator. Cells in the control group were exposed to atmospheric gas $\left(5 \% \mathrm{CO}_{2} / 21 \% \mathrm{O}_{2} /\right.$ balanced $\mathrm{N}_{2}$ ) for $8 \mathrm{~h}$. Atmospheric gas $\left(5 \% \mathrm{CO}_{2} / 21 \% \mathrm{O}_{2} /\right.$ balanced $\left.\mathrm{N}_{2}\right)$ was pumped in via a calibrated agent-specific vaporizer, as described previously (9). Gas phase concentrations in the incubator were verified by infrared absorbance of the effluent gas, and were constantly monitored and maintained at the appropriate concentrations throughout the experiments using an infrared Ohmeda 5330 Agent Monitor (Datex-Ohmeda; GE Healthcare Life Sciences, Pittsburgh, PA, USA). Since the experimental cell culture plates were inside the sealed anesthetic exposure chamber, which was continuously perfused with a constant concentration of anesthetic, the anesthetic concentration in the cell culture medium remained stable as previously reported (11). There was no evidence of anesthetic degradation in the cell culture over an $8 \mathrm{~h}$ time period. A group of cells were pretreated with xestospongin C (100 nM; Merck Millipore) for 30 in at room temperature prior to isofluarne exposure.

Imaging analysis of Annexin $V$ and propidium iodide $(P I)$. One of the early indications of cell damage is the translocation of the phospholipid phosphatidylserine from the inner to the outer leaflet of the plasma membrane. Annexin V is a phospholipid-binding protein with a high affinity for phosphatidylserine, which binds to it once exposed to environmental stress. PI is able to bind to nucleic acids following penetration of a breached plasma membrane, which occurs in the later stages of cell damage. Immediately after treatment, the cells were analyzed using an Annexin V/PI apoptosis kit [cat. no. AP 101-30; Multi Sciences (Lianke) Biotech Co., Ltd., Hangzhou, China]. A total of $1-5 \times 10^{5}$ cells were collected by centrifugation $\left(5,000 \times \mathrm{g}, 4^{\circ} \mathrm{C}, 5 \mathrm{~min}\right)$ and were resuspended in $500 \mu 11 \mathrm{X}$ binding buffer, to which was added $5 \mu \mathrm{l}$ Annexin V and $10 \mu \mathrm{l}$ PI. The cells were then incubated at room temperature for $5 \mathrm{~min}$ in the dark, and the number of Annexin V- and/or PI-positive cells were determined by flow cytometry.

Observation of the changes in cell ultrastructure. Following experimental treatment, the cells were fixed in $2.5 \%$ glutaraldehyde. Following dehydration, soaking and embedding as described previously (12), the samples were sliced and stained in order to prepare transmission electron microscopy (TEM) specimens for the observation of cell ultrastructure alterations using a Libra200 microscope (Zeiss GmbH, Jena, Germany).

Measurements of $\left.\left[\mathrm{Ca}^{2+}\right]_{c} \cdot \mathrm{CCa}^{2+}\right]_{\mathrm{c}}$ was measured by flow cytometry. Cells were washed and incubated for $24 \mathrm{~h}$ at $37^{\circ} \mathrm{C}$ in $\mathrm{NaCl}$ Ringers solution containing $1 \mathrm{mM} \mathrm{CaCl}$; or in Na-gluconate Ringers solution [125 mM Na-D-gluconate, $5 \mathrm{mM}$ K-D-gluconate, $1 \mathrm{mM} \mathrm{MgSO}_{4}, 32 \mathrm{mM}$ HEPES/NaOH (pH 7.4) and $5 \mathrm{mM}$ glucose] containing $1 \mathrm{mM}$ Ca-D-gluconate2. Cells were then loaded with Fluo-3/AM in $\mathrm{CaCl}_{2}(1 \mathrm{mM})$-containing $\mathrm{NaCl}$ or Na-gluconate Ringers solution with $2 \mu \mathrm{M}$ Fluo-3/AM. Cells were incubated at $37^{\circ} \mathrm{C}$ for $15 \mathrm{~min}$ with agitation, and were then washed twice and resuspended in $\mathrm{CaCl}_{2}(2 \mathrm{mM})$-containing $\mathrm{NaCl}$ Ringers solution. $\mathrm{Ca}^{2+}$-dependent Fluo-3/AM fluorescence intensity was then measured in fluorescence channel FL-1, which represents changes in $\left[\mathrm{Ca}^{2+}\right]_{\mathrm{c}}$.

Western blot analysis. Following treatment, the cells were harvested and total proteins were obtained by centrifugation at $10,000 \times \mathrm{g}$ for $30 \mathrm{~min}$ at $4^{\circ} \mathrm{C}$. Protein concentration was determined using a Bio-Rad Dc assay kit (Bio-Rad Laboratories, Inc., Hercules, CA, USA) and were samples were subjected to western blot analyses. Proteins (1-5 $\mu \mathrm{g} / \mathrm{ml})$ were separated on by sodium dodecyl sulfate-polyacrylamide gel electrophoresis on $7.5 \%$ gels and transferred onto polyvinylidene difluoride membranes. The membranes were blocked at room temperature for $90 \mathrm{~min}$ in Tris-buffered saline-Tween containing 5\% skim milk. Membranes were incubated with rabbit polyclonal anti-IP3R antibody (1:800; cat. no. 07-1213; EMD Millipore, Billerica, MA, USA) for $1 \mathrm{~h}$ at room temperature to detect the protein levels of IP3R $(250 \mathrm{kDa})$. Anti- $\beta$-actin antibody $(1: 10,000$; cat. no. MABE89; Sigma-Aldrich; Merck Millipore) was used to detect $\beta$-actin $(42 \mathrm{kDa})$. Subsequently, the membrane was incubated with horseradish peroxidase-conjugated secondary antibody (cat. no. AP181P; Bio-Rad Laboratories, Inc.) for 
A

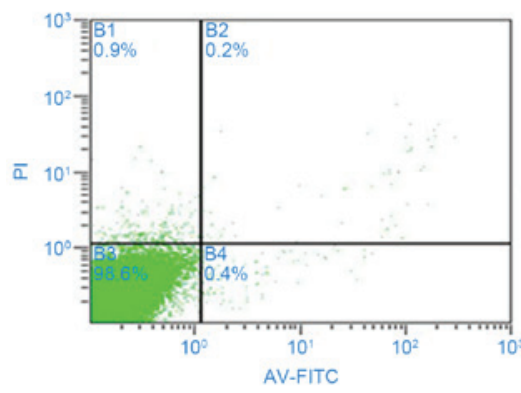

C

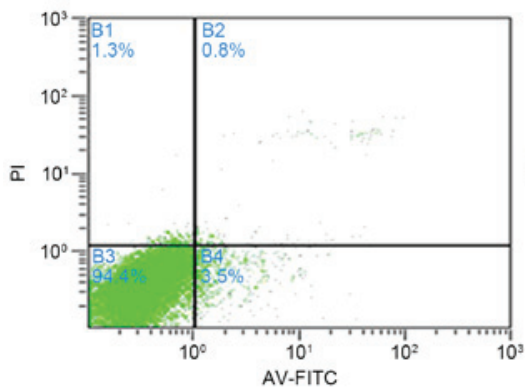

$\mathbf{E}$

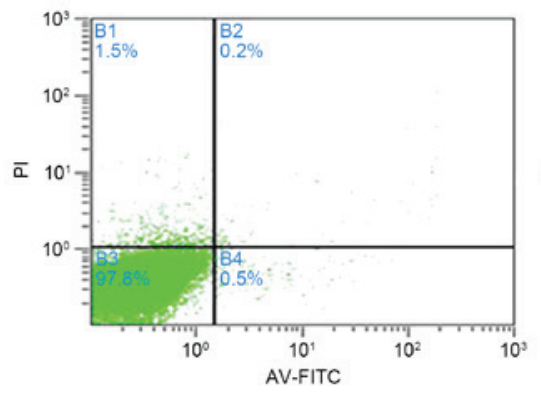

B

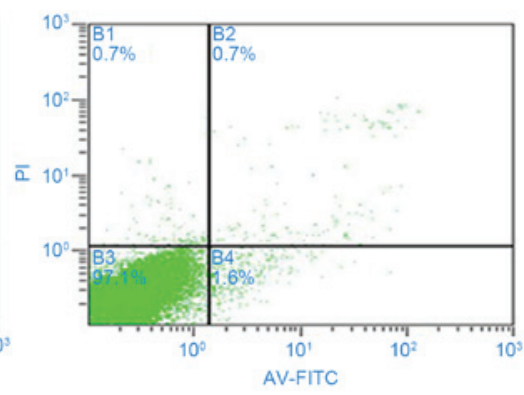

D

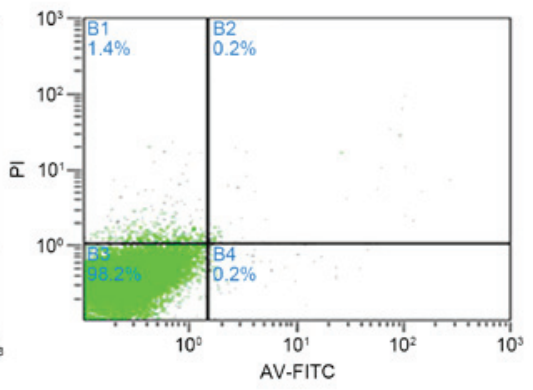

$\mathbf{F}$

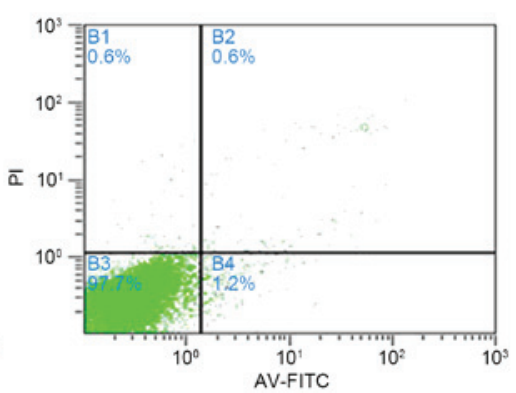

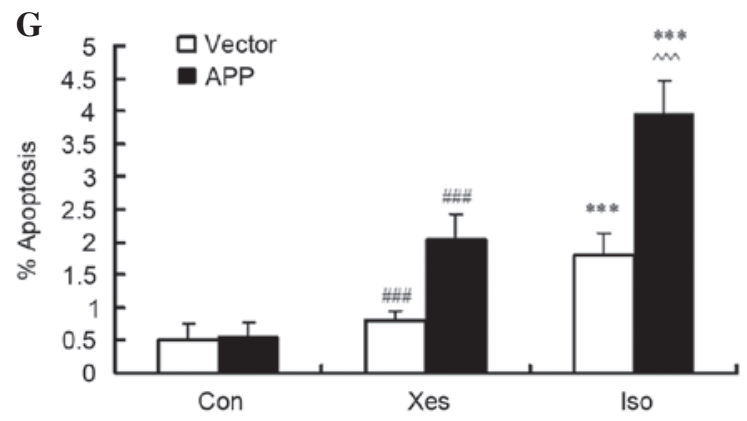

Figure 1. After cells were treated with $1.2 \%$ isoflurane for $8 \mathrm{~h}$, apoptosis was determined by flow cytometry. Briefly, the cells were harvested, stained with annexin V-fluorescein isothiocyanate (FITC) and propidium iodide (PI), and were then analyzed. (A-C) Representative results from one of six independent experiments on mutated $\beta$-amyloid precursor protein (APP)-transfected SH-SY5Y cells treated with (A) the control condition (Con), (B) xestospongin C plus isoflurane (Xes) and (C) isoflurane (Iso). (D-F) Representative results from vector-transfected SH-SY5Y cells treated with (D) Con, (E) Xes and (F) Iso. The quadrants of each plot exhibit the following: Viable cells (Annexin/PI; lower left), early apoptotic cells (Annexin ${ }^{+} / \mathrm{PI}^{-}$, lower right), necrotic cells (Annexin ${ }^{+} / \mathrm{PI}^{+}$, upper right), and late apoptotic cells (Annexin $/ \mathrm{PI}^{+}$, upper left). (G) Data are presented as a sum of the percentage of apoptotic cells in early apoptosis $\left(a_{n} \operatorname{exin}^{+} / \mathrm{PI}\right)$ and late apoptosis $\left(\operatorname{annexin}{ }^{+} / \mathrm{PI}^{+}\right)$. Data are presented as the mean \pm standard deviation of at least three separate experiments. ${ }_{* * * *} \mathrm{P}<0.001$ compared with Con and Xes; ${ }^{\# \# \#} \mathrm{P}<0.001$ compared with Con; ${ }^{\wedge \wedge} \mathrm{P}<0.001$ compared with vector control group.

$1 \mathrm{~h}$ at room temperature. The signal was visualized using a Kodak Image Station 2000R system (Kodak, Rochester, NY, USA) and RapidStep ${ }^{\mathrm{TM}}$ reagent (Merck Millipore). Each band in presented western blots represents an independent experiment. Results were averaged from between three and 10 independent experiments. Briefly, signal intensity was analyzed using the National Institutes of Health (NIH) image program (NIH Image 1.62; NIH, Bethesda, MD, USA). Western blots were semi-quantified according to two steps. Firstly, levels of $\beta$-actin were used to normalize levels of IP3R to the control, in order to account for any loading differences in total protein amount. Secondly, changes in IP3R protein levels in treated cells were presented as a percentage of those in control cells. 
Statistical analysis. Data were analyzed by SPSS 13.0 statistical software (SPSS, Inc., Chicago, IL, USA). All data met normality and homogeneity of variance, and were presented as the mean \pm standard deviation. Results regarding the effects of isoflurane on mutated APP-transfected cells and vector-transfected cells were analyzed using unpaired two-tail t-test. All other data were analyzed by one-way analysis of variance followed by Newman-Keuls multiple comparison tests. $\mathrm{P}<0.05$ was considered to indicate a statistically significant difference.

\section{Results}

Assessment of control conditions. The present study initially assessed whether control conditions $\left(5 \% \mathrm{CO}_{2} / 21 \%\right.$ $\mathrm{O}_{2}$ /balanced $\mathrm{N}_{2}$ ) were able to affect cell apoptosis, $\left[\mathrm{Ca}^{2+}\right]_{\mathrm{c}}$ and IP3R expression. There were no significant differences in cell apoptosis, $\left[\mathrm{Ca}^{2+}\right]_{\mathrm{c}}$ and IP3R levels in the cells exposed to control conditions compared with the cells exposed to standard cell incubator conditions (data not shown).

Isoflurane induces apoptosis of SH-SY5Y cells transfected with APP mutation. Our previous study demonstrated that exposure to isoflurane at $1 \mathrm{MAC}$ for $12 \mathrm{~h}$, or at $2 \mathrm{MAC}$ for $8 \mathrm{~h}$ (9), may decrease cell viability; therefore, in SH-SY5Y cells, the effects of isoflurane exposure at $1 \mathrm{MAC}$ were compared between cells transfected with an APP mutation and those transfected with the vector. In order to confirm that apoptosis observed in SH-SY5Y cells was induced by isoflurane, the number of Annexin V-positive/PI-negative and Annexin V-positive/PI-positive cells was counted following exposure to isoflurane (1 MAC) for $8 \mathrm{~h}$ (Fig. 1). Isoflurane induced apoptosis in mutated APP-transfected SH-SY5Y cells (Fig. 1A-C) and vector-transfected SH-SY5Y cells (Fig. 1D-F). As shown in Fig. 1C, treatment with 1.2\% isoflurane ( 1 MAC) for $8 \mathrm{~h}$ induced a significant increase in the apoptotic rate of mutated APP-transfected SH-SY5Y cells compared with the vector-transfected SH-SY5Y cells (Fig. 1F). Inhibition of IP3R activity with xestospongin $\mathrm{C}$ partly reduced isoflurane-induced cell apoptosis (Fig. 1B and E).

Effects of isoflurane on the ultrastructure of mutated APP-transfected SH-SY5Y cells. The results of a TEM analysis (Fig. 2) indicated that vector-transfected SH-SY5Y cells were characterized by a smooth nuclear membrane, and slight expansion and degranulation of the ER. Conversely, mutated APP-transfected SH-SY5Y cells exhibited marked morphological alterations; there were a large number of vacuoles in the cytoplasm, organelle structure was incomplete, the ER exhibited a moderate to high degree of swelling and marked degranulation, mitochondrial cristae were disordered, the membrane structure was damaged, and the number of microtubules was increased.

Effects of isoflurane on intracellular calcium homeostasis. $\left[\mathrm{Ca}^{2+}\right]_{\mathrm{c}}$ was determined by measuring the average value of calcium fluorescence intensity by flow cytometry in mutated APP-transfected SH-SY5Y cells (Fig. 3A-C) and vector-transfected SH-SY5Y cells (Fig. 3D-F). Treatment with isoflurane (1 MAC) induced a significant elevation in the $\left[\mathrm{Ca}^{2+}\right]_{\mathrm{c}}$ of mutated APP-transfected SH-SY5Y cells (Fig. 3C) compared with in
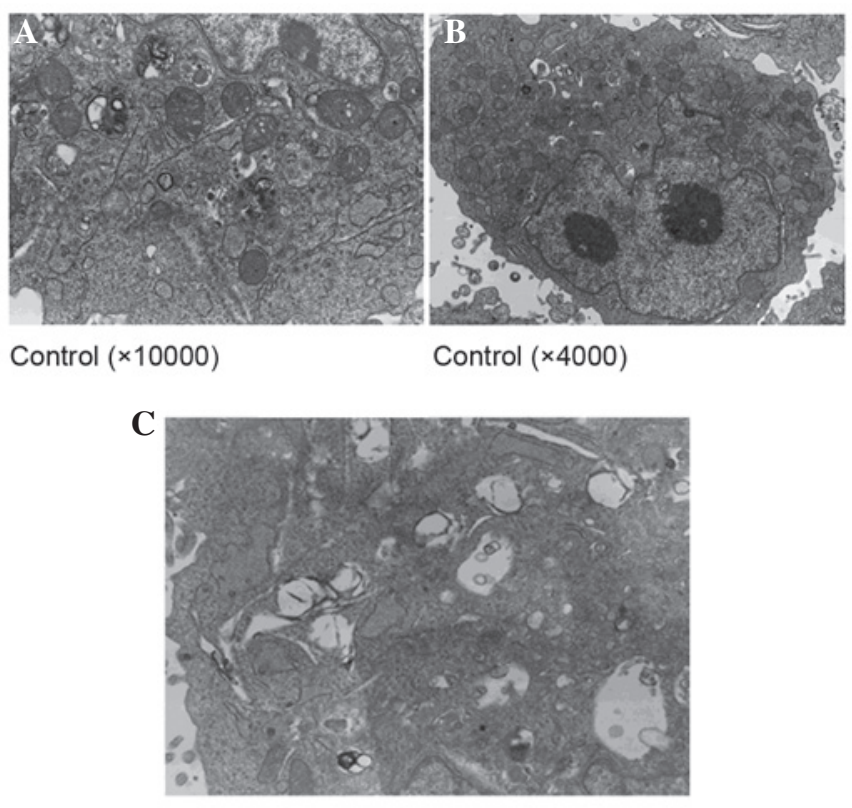

Isoflurane $(\times 10000)$

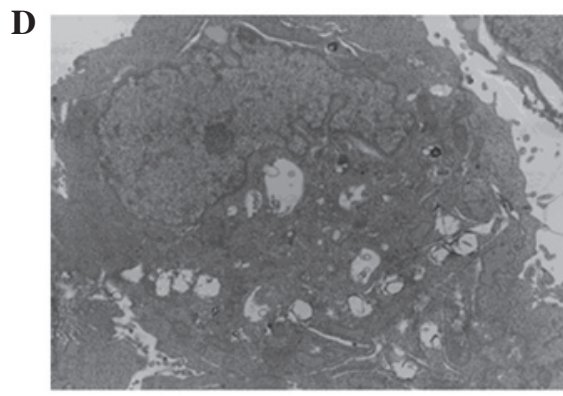

Isoflurane $(\times 5000)$

Figure 2. Transmission electron microscopy of APP-transfected SH-SY5Y cells. (A and B) Cells in the control group exhibited the following characteristics: Smooth nuclear membrane, and slight expansion and degranulation of the endoplasmic reticulum (ER). (C and D) Characteristics of the cells in the isoflurane group were as follows: Large number of vacuoles in the cytoplasm, incomplete organelle structure, a moderate to high degree of ER swelling, ER degranulation, disordered mitochondrial cristae, damaged membrane structure, and microtubule microfilament contents increased. These ultrastructural changes indicated that exposure to $1 \mathrm{MAC}$ isoflurane for $8 \mathrm{~h}$ could induce cell injury.

the vector-transfected SH-SY5Y cells (Fig. 3F). In addition, it was determined whether calcium release from the ER via IP3R contributed to isoflurane-induced elevation in the $\left[\mathrm{Ca}^{2+}\right]_{\mathrm{c}}$ of mutated APP-transfected SH-SY5Y cells. Pretreatment with the potent IP3R antagonist, xestospongin $\mathrm{C}$, for 30 min reduced isoflurane-induced calcium release from the ER (Fig. 3B). These results suggest that exposure to $1 \mathrm{MAC}$ isoflurane for $8 \mathrm{~h}$ may significantly increase intracellular calcium concentration via activation of IP3R in mutated APP-transfected SH-SY5Y cells.

Isoflurane increases the protein expression levels of IP3R. The present study assessed the effects of $1.2 \%$ isoflurane on IP3R expression in SH-SY5Y cells. Treatment with $1.2 \%$ isoflurane led to increases in the protein expression levels of IP3R in mutated APP- and vector-transfected SH-SY5Y cells compared with the cells in the control or xestospongin $\mathrm{C}$ groups (Fig. 4A and B). Compared with vector-transfected SH-SY5Y cells, IP3R expression was increased in mutated 
A

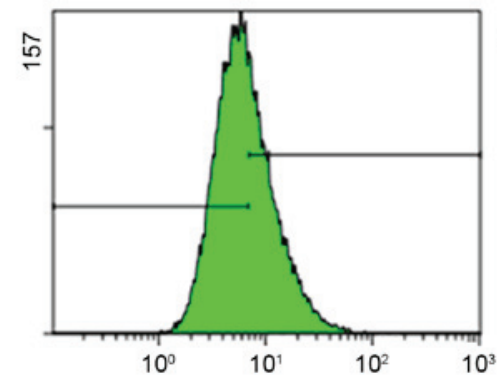

C

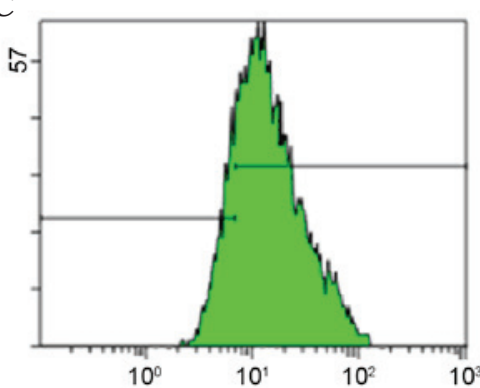

E

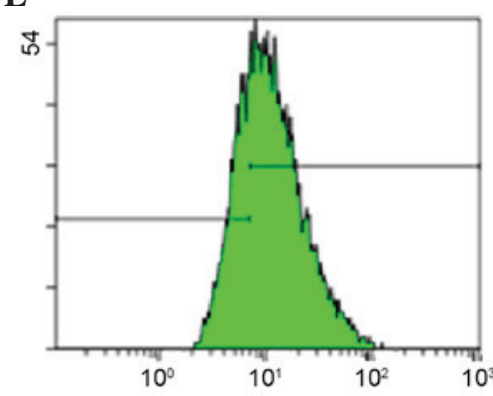

B

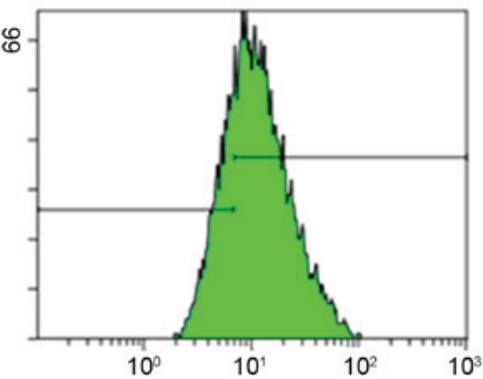

D

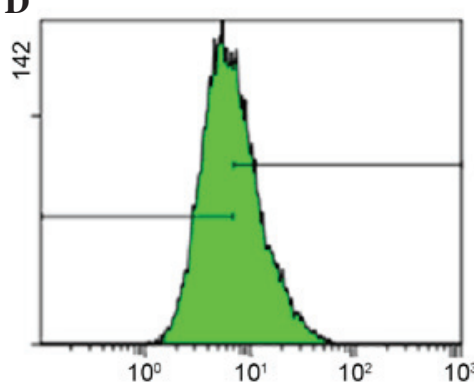

F

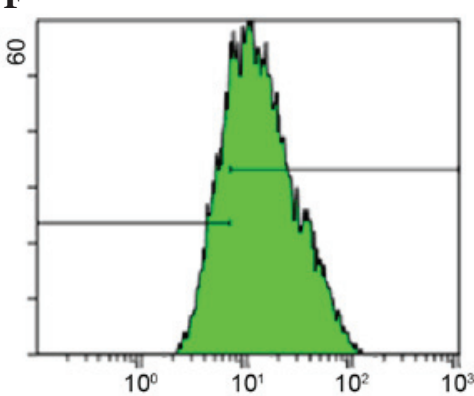

G

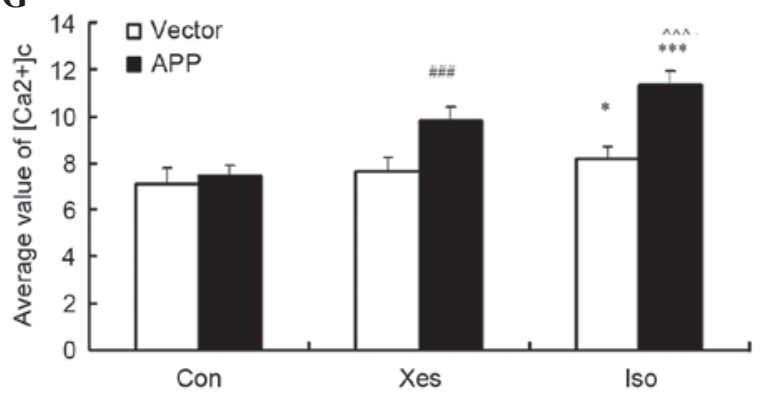

Figure 3. After cells were treated with $1.2 \%$ isoflurane for $8 \mathrm{~h}$, cytosolic calcium concentration $\left(\left[\mathrm{Ca}^{2+}\right]_{\mathrm{c}}\right)$ was determined by flow cytometry. Briefly, the cells were harvested, stained with Fluo-3/AM and were then analyzed. (A-C) Representative results from one of six independent experiments on mutated $\beta$-amyloid precursor protein (APP)-transfected SH-SY5Y cells treated with (A) the control condition (Con), (B) xestospongin C plus isoflurane (Xes) and (C) isoflurane (Iso). (D-F) Representative results from vector-transfected SH-SY5Y cells treated with (D) Con, (E) Xes and (F) Iso. The positive rate of $\left[\mathrm{Ca}^{2+}\right]_{\mathrm{c}}$ loading with Fluo-3/AM was $>99 \%$. (A-F) X-axis refers to the average value of calcium fluorescence intensity, and Y-axis refers to cell count. (G) Data are presented as the mean \pm standard deviation of at least three separate experiments. ${ }^{~} \mathrm{P}<0.05$ and ${ }^{* * * *} \mathrm{P}<0.001$ compared with Con and Xes; ${ }^{\# \# \#} \mathrm{P}<0.001$ compared with Con; ${ }^{\wedge \wedge} \mathrm{P}<0.001$ compared with vector control group.

APP-transfected SH-SY5Y cells. These results indicate that isoflurane exposure at $1 \mathrm{MAC}$ for $8 \mathrm{~h}$ may increase IP3R protein expression, and $100 \mathrm{nM}$ xestospongin $\mathrm{C}$ could partly suppress isoflurane-induced upregulation of IP3R protein expression.

\section{Discussion}

The present study demonstrated that treatment with isoflurane at equipotent concentrations induced apoptosis of SH-SY5Y cells by elevating $\left[\mathrm{Ca}^{2+}\right]_{\mathrm{c}}$ levels, whereas exposure to control conditions $\left(5 \% \mathrm{CO}_{2} / 21 \% \quad \mathrm{O}_{2} / \mathrm{N}_{2}\right)$ did not. Furthermore, isoflurane induced a larger increase in apoptotic rate, and increased the elevation of $\left[\mathrm{Ca}^{2+}\right]_{\mathrm{c}}$ and IP3R protein levels in mutated APP-transfected SH-SY5Y cells compared with in vector-transfected control cells. These findings implicated IP3R as the main source of calcium release from the ER.

Previous studies have proposed that inhalational anesthetics induce apoptosis via dysregulated intracellular 
A

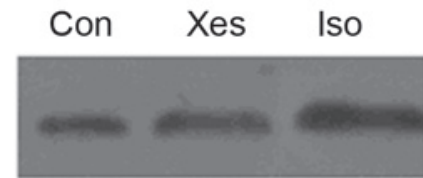

$\beta$-actin

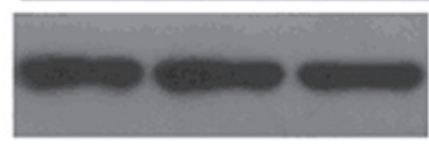

B
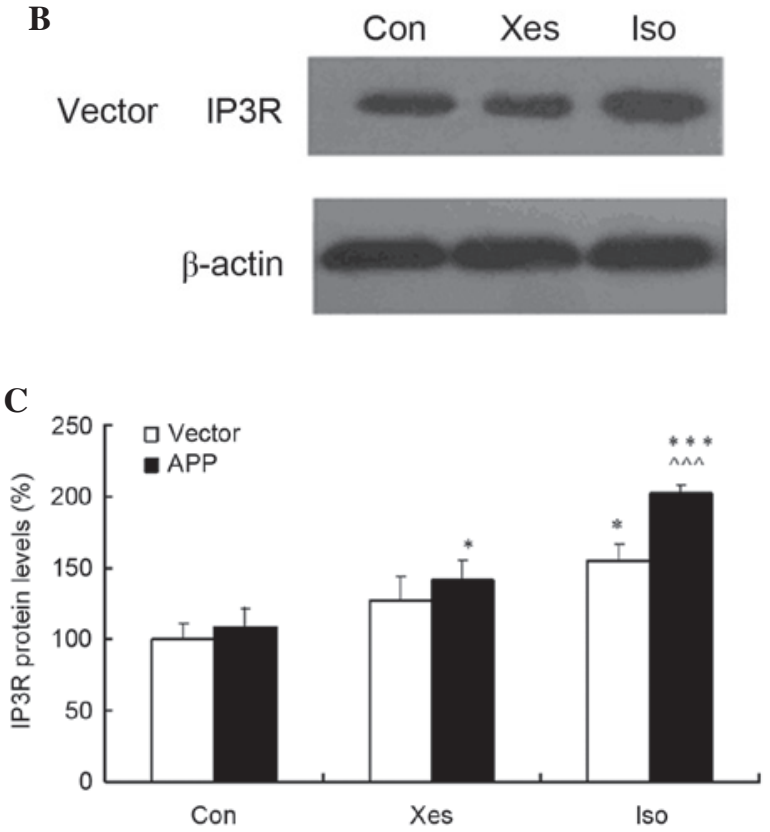

Figure 4. Isoflurane induces inositol 1,4,5-trisphosphate receptor (IP3R) activation in mutated $\beta$-amyloid precursor protein (APP)-transfected SH-SY5Y cells. (A and B) Treatment with 1.2\% isoflurane (Iso) inducesd IP3R cleavage compared with in the control (Con) and xestospongin C (Xes) groups in both cell types. There was no significant difference in $\beta$-actin expression between the groups. (C) Treatment with $1.2 \%$ isoflurane induced a higher increase in IP3R protein expression in the mutated APP-transfected SH-SY5Y cells compared with vector-transfected SH-SY5Y cells. Data are presented as the mean \pm standard deviation of at least three separate experiments. ${ }^{*} \mathrm{P}<0.05$ compared with Con. ${ }^{* * *} \mathrm{P}<0.001$ compared with $\mathrm{Con}$ and Xes. ${ }^{\wedge \wedge} \mathrm{P}<0.001$ compared with vector control group.

calcium homeostasis $(5,7,13) . \mathrm{Ca}^{2+}$ regulation in neurons is complex. $\left[\mathrm{Ca}^{2+}\right]_{c}$ in neurons is maintained at $-100 \mathrm{nM}$, a low level relative to the extracellular fluid (extracellular $\left.\left[\mathrm{Ca}^{2+}\right]=1.2 \mathrm{mM}\right)$. The dynamic balance of intracellular $\mathrm{Ca}^{2+}$ homeostasis is maintained by $\mathrm{Ca}^{2+}$ transportation across cell membranes and regulation of intracellular calcium stores. In response to stress, neurons will instantly improve $\left[\mathrm{Ca}^{2+}\right]_{\mathrm{c}}$ levels, in order to trigger a series of physiological activities. The ER is the primary source of releasable intracellular calcium in neurons (11) and has an important role in the maintenance of intracellular calcium homeostasis, protein synthesis, cell survival and apoptosis $(14,15)$. RyR and IP3R are calcium-activated calcium release channels that are present on the ER membrane. Calcium release from the ER via RyR activation can result in activation of IP3R and vice versa. In neurons, isoflurane appears to induce calcium release from the ER; however, it remains unclear whether this is due to direct or indirect effects on IP3 or RyR. Our previously published and current data suggested that overactivation of
IP3R may contribute to isoflurane-induced calcium elevation and cell apoptosis. Excessive calcium release from the ER via IP3R may induce calcium overload in the mitochondria and depletion of ER calcium (11), which may result in collapse of the mitochondrial membrane potential and promotion of apoptosis.

To determine the importance of IP3R in isoflurane-induced apoptosis, IP3R activity can be altered either genetically or with the use of pharmacological agents. A previous study in cultured chicken $\mathrm{T}$ lymphocytes with triple knock out of IP3R indicated that the cells were resistant to inhalational anesthetic-induced apoptosis, decreases in ER calcium concentrations and increases in cytosolic and mitochondrial calcium concentrations $(5,7)$. Furthermore, rat pheochromocytoma neurosecretory PC12 cells with elevated IP3R activity that were transfected with presenilin-1 (L286V), or Q-111 rat striatal neurons (a cell model of Huntington disease), were susceptible to isoflurane-induced apoptosis and ER calcium release. However, these effects were significantly attenuated following treatment with the IP3R antagonist xestospongin C (5). These studies suggested that activation of IP3R may have an important role in inhalational anesthetic toxicity. In support of this viewpoint, the present data clearly indicated that prolonged isoflurane exposure was able to induce cell apoptosis via direct activation of IP3R, and treatment with the IP3R antagonist xestospongin $C$ reduced the rate of cell apoptosis. Based on these findings, we aim to develop a therapeutic approach that targets IP3R to protect patients undergoing inhalational anesthesia from the potential deleterious side effects of prolonged exposure.

The present study demonstrated that isoflurane-induced enhancement in $\left[\mathrm{Ca}^{2+}\right]_{c}$ in cells carrying an APP mutation is consistent with the higher degree of neurotoxicity observed in these same cells after exposure to isoflurane. These data indicated that a risk factor for early AD may increase cell susceptibility to clinical concentrations of isoflurane. A previous study reported that a clinically relevant concentration of isoflurane was able to induce apoptosis, alter APP processing, and increase $A \beta$ levels in $\mathrm{H} 4$ human neuroglioma cells stably transfected with an APP mutation, which is thought to be a key feature in the pathogenesis of AD (16). $\mathrm{A} \beta$ has been demonstrated to augment calcium release from the ER via RyR or IP3R; therefore, anesthetic-induced increases in $A \beta$ production may be considered another indirect mechanism by which inhalational anesthetics enhance ER calcium release (17). Isoflurane induces apoptosis via calcium release from the ER, which consequently increases the activity of beta-site APP-cleaving enzyme and $\gamma$-secretase, which are associated with the generation of $A \beta$ proteins (18). Isoflurane-mediated elevation and aggregation of $A \beta$ proteins may further induce apoptosis, resulting in the initiation of a vicious cycle. However, due to the findings of xestospongin $\mathrm{C}$ experiments in PC12 cells and rat cerebral cortical neurons it is likely that isoflurane acts directly on IP3R to enhance calcium release.

In the present study, ultrastructural alterations to cells following exposure to isoflurane were determined by TEM; the results demonstrated that the most typical changes were swelling of the mitochondria and the ER. Abnormal changes in ER structure can induce calcium flux into the cytoplasm, 
resulting in a rapid increase in intracellular calcium concentration and promotion of cell apoptosis. Previous studies have proposed that ER-induced apoptosis ultimately occurs via the mitochondrial pathway $(19,20)$. Abnormal alterations in the mitochondria may be caused by changes to the ER. In addition, microtubule and microfilament content increased following isoflurane exposure; microtubules are an important component of the cytoskeleton, which are associated with mitosis, intracellular translocation, overall cellular morphology, cell markers, and various other functions. The structural integrity of microtubules is the basis of nutrient transport between the nerve cell body and axons. Several pathological clinical studies demonstrated that neuritic plaques and hippocampal neurofibrillary tangles are associated with dementia severity $(21,22)$. In the present study typical apoptotic bodies were not detected by TEM, suggesting that apoptosis may occur at an early stage.

It has long been reported that anesthetics enhance calcium release via the activation of RyR, which is the other major calcium release channel complex on the ER (23). Similar to IP3R, RyR has an important role in normal cell function and various neurodegenerative diseases. Since IP3R and RyR interact, it remains unclear as to whether one or both of these receptors are direct targets of isoflurane; however, calcium influx from the extracellular space also has a role in isoflurane cytotoxicity (13). Memantine is a noncompetitive partial antagonist of N-methyl-D-aspartate receptor (NMDAR), which inhibits calcium influx and markedly suppresses isoflurane-induced apoptosis and cell death (24). Further studies are required to investigate how calcium release from the ER and calcium influx from the extracellular space may contribute to anesthetic-associated toxicity.

The present study only focused on an APP mutation; however, there are other factors that contribute to neurodegeneration in AD. These include tau, presenilin, various secretases, apolipoprotein E, and perhaps heat shock proteins and ferritins. Several of these factors may be modulated by calcium (25-27).

A presenilin-1 mutation has been reported to render neurons susceptible to isoflurane toxicity by inducing abnormal calcium release from the ER via activation of IP3R (28). The measurements of intracellular calcium conducted in the present study were limited to the cytosolic compartment; therefore, secondary or primary alterations in $\mathrm{Ca}^{2+}$ levels may be occurring in the ER and mitochondria. Further studies are required to clarify the effects of volatile anesthetics on calcium dynamics in these organelles.

SH-SY5Y cells are not neurons; therefore, their sensitivity to isoflurane exposure may differ, and the concentration and duration of isoflurane exposure required to induce cell apoptosis may be different. The results of the present study were from cultured cell lines, more studies are required in animals, such as rodents and primates, to investigate the effects of isoflurane exposure on animal memory, cognition and behavior.

In conclusion, the present study demonstrated that an APP mutation associated with familial AD may render SH-SY5Y cells more vulnerable to isoflurane-induced cytotoxicity. Calcium release from IP3R on the ER may underlie the cytotoxic effects of isoflurane. Notably, pharmacological inhibition of IP3R attenuated isoflurane-induced cell apoptosis. Further investigation into the cytotoxic effects of isoflurane is required in animal models and in patients with risk factors for, or symptoms of AD. These findings may improve the decision-making capabilities of anesthesiologists with regards to the use of inhalational anesthetics in the elderly population.

\section{References}

1. Bekker AY and Weeks EJ: Cognitive function after anesthesia in the elderly. Best Pract Res Clin Anaesthesiol 17: 259-272, 2003.

2. Moller JT, Cluitmans P, Rasmussen LS, Houx P, Rasmussen H, Canet J, Rabbitt P, Jolles J, Larsen K, Hanning CD, et al: Long-term postoperative cognitive dysfunction in the elderly ISPOCD1 study. ISPOCD investigators. International Study of Post-Operative Cognitive Dysfunction. Lancet 351: 857-861, 1998.

3. Newman MF, Kirchner JL, Phillips-Bute B, Gaver V, Grocott H, Jones RH, Mark DB, Reves JG and Blumenthal JA; Neurological Outcome Research Group and the Cardiothoracic Anesthesiology Research Endeavors Investigators: Longitudinal assessment of neurocognitive function after coronary-artery bypass surgery. $\mathrm{N}$ Engl J Med 344: 395-402, 2001.

4. Wei H and Xie Z: Anesthesia, calcium homeostasis and Alzheimer's disease. Curr Alzheimer Res 6: 30-35, 2009.

5. Jevtovic-Todorovic V, Hartman RE, Izumi Y, Benshoff ND, Dikranian K, Zorumski CF, Olney JW and Wozniak DF: Early exposure to common anesthetic agents causes widespread neurodegeneration in the developing rat brain and persistent learning deficits. J Neurosci 23: 876-882, 2003.

6. Culley DJ, Baxter MG, Yukhananov R and Crosby G: Long-term impairment of acquisition of a spatial memory task following isoflurane-nitrous oxide anesthesia in rats. Anesthesiology 100: 309-314, 2004.

7. Culley DJ, Baxter M, Yukhananov R and Crosby G: The memory effects of general anesthesia persist for weeks in young and aged rats. Anesth Analg 96: 1004-1009, 2003.

8. Bianchi SL, Tran T, Liu C, Lin S, Li Y, Keller JM, Eckenhoff RG and Eckenhoff MF: Brain and behavior changes in 12-month-old $\mathrm{Tg} 2576$ and nontransgenic mice exposed to anesthetics. Neurobiol Aging 29: 1002-1010, 2008

9. Wang QJ, Wang XL, Zhao J, Zhao ZJ, Lv YX and Zhu HX: Effects of different concentrations of isoflurane on viability in rat primary cortical neurons. Chin J Anesthesiol 30: 673-675, 2010.

10. Luciani DS, Gwiazda KS, Yang TL, Kalynyak TB, Bychkivska Y, Frey MH, Jeffrey KD, Sampaio AV, Underhill TM and Johnson JD: Roles of $\mathrm{IP}_{3} \mathrm{R}$ and $\mathrm{RyR} \mathrm{Ca}^{2+}$ channels in endoplasmic reticulum stress and beta-Cell death. Diabetes 58: 422-432, 2009.

11. Samtleben S, Wachter B and Blum R: Store-operated calcium entry compensates fast ER calcium loss in resting hippocampal neurons. Cell Calcium 58: 147-159, 2015.

12. de Carvalho ND, Garcia CT, Ferreira AK, Batista DR, Cassola AC, Maria D, Lebrun I, Carneiro SM, Afeche SC, Marcourakis T and Sandoval MR: Neurotoxicity of coral snake phospholipases A2 in cultured rat hippocampal neurons. Brain Res 1552:1-16, 2014.

13. Geiger JE, Hickey $\mathrm{CM}$ and Magoski NS: $\mathrm{Ca}_{2}^{+}$entry through a non-selective cation channel in Aplysia bag cell neurons. Neuroscience 162: 1023-1038, 2009.

14. Gallego-Sandín S, Alonso MT and García-Sancho J: Calcium homoeostasis modulator 1 (CALHM1) reduces the calcium content of the endoplasmic reticulum (ER) and triggers ER stress. Biochem J 437: 469-475, 2011.

15. Kim HR, Kim MS, Kwon DY, Chae SW and Chae HJ: Bosellia serrata-induced apoptosis is related with ER stress and calcium release. Genes Nutr 2: 371-374, 2008.

16. Xie Z, Dong Y, Maeda U, Alfille P, Culley DJ, Crosby G and Tanzi RE: The common inhalation anesthetic isoflurane induces apoptosis and increases amyloid beta protein levels. Anesthesiology 104: 988-994, 2006.

17. Ferreira IL, Ferreiro E, Schmidt J, Cardoso M, Pereira CM, Carvalho AL, Oliveira CR and Rego AC: A $\beta$ and NMDAR activation cause mitochondrial dysfunction involving ER calcium release. Neurobiol Aging 36: 680-692, 2015.

18. Zhao Y, Liang G, Chen Q, Joseph DJ, Meng Q, Eckenhoff RG, Eckenhoff MF and Wei $\mathrm{H}$ : Anesthetic induced neurodegeneration mediated via inositol 1,4,5-trisphosphate receptors. J Pharmacol Exp Ther 333: 14-22, 2010. 
19. Timmins JM, Ozcan L, Seimon TA, Li G, Malagelada C Backs J, Backs T, Bassel-Duby R, Olson EN, Aderson ME and Tabas I: Calcium/calmodulin-dependent protein kinase II links ER stress with Fas and mitochondrial apoptosis pathways. J Clin Invest 119: 2925-2941, 2009.

20. Su TR, Tsai FJ, Lin JJ, Huang HH, Chiu CC, Su JH, Yang YT, Chen JY, Wong BS and Wu YJ: Induction of apoptosis by 11-dehydrosinulariolide via mitochondrial dysregulation and ER stress pathways in human melanoma cells. Mar Drugs 10: 1883-1898, 2012.

21. Presti MF, Schmeichel AM, Low PA, Parisi JE and Benarroch EE: Degeneration of brainstem respiratory neurons in dementia with Lewy bodies. Sleep 37: 373-378, 2014.

22. Thomas T, Miners S and Love S: Post-mortem assessment of hypoperfusion of cerebral cortex in Alzheimer's disease and vascular dementia. Brain 138: 1059-1069, 2015.

23. Gordienko DV and Bolton TB: Crosstalk between ryanodine receptors and $\mathrm{IP}(3)$ receptors as a factor shaping spontaneous $\mathrm{Ca}(2+)$-release events in rabbit portal vein myocytes. J Phsyiol 542: 743-762, 2002.
24. Zhang GH, Dong YL, Zhang B, Ichinose F, Xu Wu, Culley DJ, Crosby G, TanziR E and Xie Z: Isoflurane-induced caspase-3 activation is dependent on cytosolic calcium and can be attenuated bymemantine. J Neurosci 28: 4551-4560, 2008.

25. Karch CM, Jeng AT and Goate AM: Calcium phosphatase calcineurin influences tau metabolism. Neurobio Aging 34: 374-386, 2013.

26. Sepulveda-Falla D, Barrera-Ocampo A, Hagel C, Korwitz A, Vinueza-Veloz MF, Zhou K, Schonewille M, Zhou H, Velazquez-Perez L, Rodriguez-Labrada R, et al: Familial Alzheimer's disease-associated presenilin-1 alters cerebellar activity and calcium homeostasis. J Clin Invest 124: 1552-1567, 2014.

27. Yefimova MG, Shcherbakova IS and Shushakova ND: Transferrin and ferritin modulate the activity of brain calcium-calmodulin-dependent phosphodiesterase. Biochemistry (Mosc) 62: 165-170, 1997.

28. Liang G, Wang Q, Li Y, Kang B, Eckenhoff MF, Eckenhoff RG and Wei $\mathrm{H}$ : A presenilin-1 mutation renders neurons vulnerable to isoflurane toxicity. Anesth and Analg 106: 492-500, 2008. 